Proteomics

\section{Structure and Function}

\author{
Tomi K. Sawyer \\ ARIAD Pharmaceuticals, Cambridge, MA, USA
}

The interdisciplinary field of proteomics has evolved over the last two decades from its conceptual origins with the display of large numbers of proteins from a specific cell line or organism using 2-D gel electrophoresis $(1,42)$. A contemporary definition of proteomics includes the large-scale identification and characterization of the proteome (complete set of proteins encoded by the genome). Characterization of the proteome includes regulation of gene expression, protein synthesis and post-translational modifications, as well as the complex structure-function relationships of intracellular and analysis of secreted proteins $(11,33)$. Following recent achievements in mapping the human genome by the Human Genome Project International Consortium (22) and Celera Genomics (39), the challenge is to understand the human proteome and further exploit the estimated 30000 human genes. Proteomics will enable global analysis of proteins, including differential display of gene expression levels in a regulated manner (e.g., disease-specific) and structure-function mapping of protein-protein interactions (e.g., catalytic or non-catalytic).

\section{Major Technology Platforms for Proteomics}

Some of the major technology platforms that have been developed for proteomics include 2-D gel electrophoresis, mass spectrometry, chip-based microassays, two-hybrid assays, phage display assays, and X-ray/ NMR analysis of protein 3-D structure (see diagram).

Historically, 2-D gel electrophoresis provided the first major technology that illustrated the potential for cataloging expressed proteins in databases $(10,32)$. It is the most widely used high-resolution technology for separating proteins based on differences in molecular charge and mass. Enhancements of gel electrophoresis (1-D and 2D) using affinity-based purification strategies are advancing the global analysis of complex protein mixtures (33).

Mass spectrometry has emerged more recently as a key technology for the identification and quantification of proteins in complex mixtures $(16,17)$. It provides sensitivity and high throughput by two principal approaches: $(i)$ mass mapping or fingerprinting of peptide fragments using matrix-assisted laser desorption/ionization (MALDI) $(4,20)$ and (ii) tandem mass spectrometry of peptide fragments to obtain specific sequence information using electrospray ionization, as exemplified by the recent development of MALDI quadrapole time-of-flight mass spectrometry
(37). Such mass spectrometry approaches use gel electrophoretic or enzymatic (proteolytic) separations to analyze proteins. A noteworthy advance in the quantitative analysis of proteins in crude mixtures without separation has been isotope-coded affinity tagging (18).

Chip-based microarray technologies have been developed to separate proteins based on surface chemistries or known protein ligands (e.g., antibodies) with subsequent identification by mass spectrometry $(7,25)$. Other promising applications of protein chip microarrays have been differential profiling (26) and high-throughput functional analysis (13).

Complementing mass spectrometry, the yeast two-hybrid system has contributed importantly to the functional characterization of protein-protein interactions (12). The yeast two-hybrid assay is based on the specific binding of a protein-DNA binding domain fusion construct to a cognate protein-DNA activation domain fusion construct (via molecular recognition between the protein components) to increase transcription of a targeted reporter gene (via proximity of the DNA binding and activation domains). The yeast two-hybrid system has been exploited for the identification of protein-protein interactions in $C$. elegans (41), bacteriophage T7 (3), vaccinia virus (28), and $S$. cerevisiae (38). Recently, a reverse two-hybrid approach has been developed to identify peptides or other compounds that disrupt protein-protein interactions (40).

Phage display can be used for large-scale analysis of protein molecular interactions based on immobilization of a "bait" protein and capture of phage particles displaying the interacting protein. Numerous applications of phage libraries exist for large-scale analysis of enzymesubstrate, protein-protein, and antibody-antigen interactions $(21,24)$.

Structural analysis of proteins by X-ray crystallography and/or NMR spectroscopy is essential to provide 3-D molecular details of protein binding domains and/or catalytic sites to understand the specificity and mechanistic properties of newly emerging therapeutic targets (8). High-throughput 3-D structure determination of new proteins by X-ray crystallography has led to advances in robust recombinant systems to produce selenomethionyl proteins, systematic and microscalable crystallization screening methods, synchrotron beam line technologies, and related methods ranging from cryo-crystallography to computerized generation of protein structures from multi-wavelength anomalous diffraction phasing data (30). Protein NMR spectroscopy plays a particularly important role in elucidating high-resolution structures of proteins (within certain size limits) and protein domains, including chemical-shift perturbation data to study ligand-protein interactions and data on internal structural dynamics from nuclear relaxation studies. Some key advances that have accelerated the development of NMR technologies include high-level protein purification systems for biosynthetic isotope enrichment, software for analysis of 3-D structures from NMR data, and more powerful magnets, superconducting probes, and other factors to significantly increase NMR sensitivity (43). 


\section{Structure-Function Mapping of Protein Molecular Interactions}

The human proteome undergoes dynamic changes in response to the regulation of gene expression and protein synthesis, post-translational modification, proteolysis, recycling, and sequestration of cellular proteins. For secreted proteins, other regulatory mechanisms affect their roles in various endocrine, metabolic, immune, or other complex biological processes. In this context, proteomics may be viewed as providing a structure-function map of protein molecular interactions (e.g., intracellular and extracellular). However, such structure-function analyses of the human proteome remain a relatively new endeavor. Recently, a Human Proteomics Initiative has been launched by the European Bioinformatics Institute and the Swiss Institute of Bioinformatics as a new collaborative endeavor to annotate, describe, and distribute a large amount of highly curated protein data related to the human proteome to the life science community (31).

Thousands of new proteins have been predicted from the human genome $(22,39)$. These exemplify a wide scope of functional properties, including metabolism, signal transduction, gene replication and transcription, protein synthesis and post-translational modification, secretion, and cytoskeletal structure. Among these properties, the post-translational modification of proteins plays a pivotal role in affecting protein molecular interactions. Some examples of post-translational modifications include phosphorylation, sulfation, glycosylation, ubiquitinylation, acetylation, and prenylation. Such structural modifications significantly alter a protein's functional properties, stability, localization, and/or secretion. Noteworthy in this regard is protein phosphorylation (at Tyr and/or Ser/Thr residues), which has become a major focus of proteomic studies directed at probing signal transduction pathways and deciphering therapeutic targets $(6,35,36)$. From a proteomics perspective, the detection and study of protein phosphorylation may be accomplished by various approaches, including mass spectrometry, metal binding, specific antibodies, and/or dephosphoryaltion by phosphatases. Protein-protein interactions, which often involve post-translational modification of one of the cognate proteins, affect many intracellular processes, especially those involving signal transduction $(27,29,34)$. Databases of these interactions are being developed $(2,9)$.

The structure-function mapping of molecular interactions of receptors, proteases, and a vast number of other catalytic proteins are likewise involved with cellular function, proliferation, differentiation, and survival and will provide the basis of significant future efforts to unravel and exploit the human proteome.

\section{Impact of Proteomics in Drug Discovery}

The integration of proteomics with functional genomics (19) and informatics (5) provides the opportunity to identify, characterize, and validate therapeutic targets. The impact of proteomics will be profound in deciphering thousands of these targets. Such validation will involve the differential display of healthy and diseased human tissues and the comparison among human, animal, and microorganisms. In addition, proteomics may provide insight to our understanding of complex diseases [several dysfunctional genes and/or protein pathways (23)] and potential opportunities for novel therapy (e.g., gene- or protein-targeted). Vaccine development may also be positively

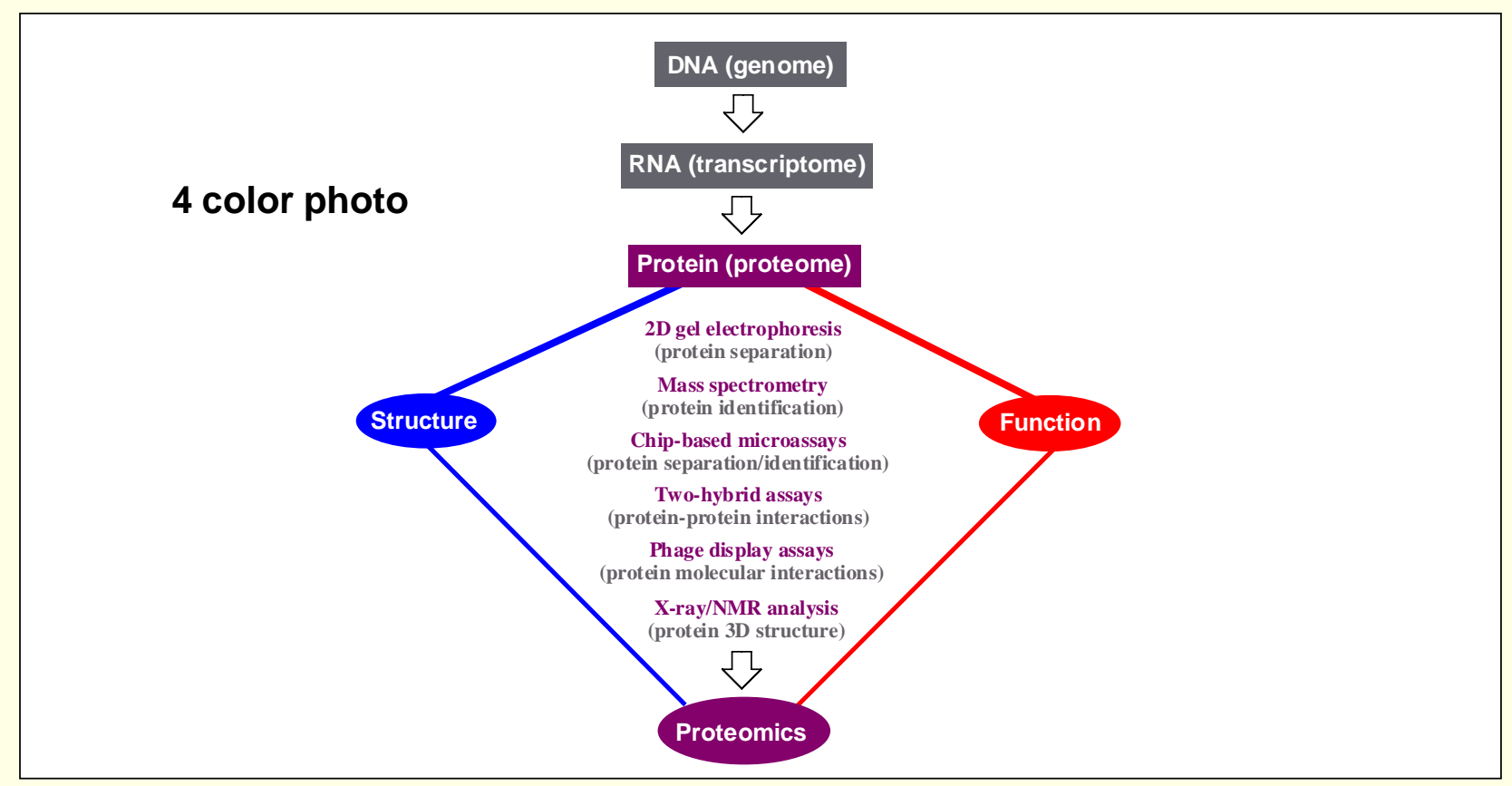

Figure 1. Some major proteomics technologies for the global analysis of protein structure and function. 
impacted by proteomics (15). Furthermore, proteomics may provide the identification of therapeutically important biomarkers for drug efficacy and/or safety studies (14). Collectively, the technology platforms for proteomics are being further refined to accelerate the drug discovery process. The next great milestone in the journey of molecular medicine and drug discovery will be the structure and function of the human proteome. This advance will represent a more complicated "blueprint" of life, and we can confidently expect that it will serve as a mind-boggling switchboard of circuitry and molecular communications.

\section{REFERENCES}

1.Anderson, N.G. and N.I. Anderson. 1996. Twenty years of two-dimensional electrophoresis: past, present and future. Electrophoresis 17:443-453.

2.Bader, G.D., I. Donaldson, C. Wolting, B.F. Ouellette, T. Pawson, and C.W.V. Hogue. 2001. BIND-the biomolecular interaction network database. Nucleic Acids Res. 29:242-245.

3.Bartel, P.L., J.A. Roecklein, D. SenGupta, and S. Fields. 1996. A protein linkage map of Escherichia coli bacteriophage T7. Nat. Genet. 12:72-77.

4.Berndt, P., U. Hobohm, and H. Langen. 1999. Reliable automatic protein identification from matrix-assisted laser desorption/ionizaiton mass spectrometric peptide fingerprints. Electrophoresis 20:3521-3526.

5.Birney, W., A. Bateman, M.E. Clamp, and T.J. Hubbard. 2001. Mining the draft human genome. Nature 409:827-828

6.Blume-Jensen, P. and T. Hunter. 2001. Oncogenic kinase signalling. Nature 411:355-365.

7.Borrebaeck, C.A.K. 2000. Antibodies in diagnostics: from immunoassays to protein chips. Immunol. Today 21:379-382.

8.Burley, S.K., S.C. Almo, J.B. Bonanno, M. Capel, M.R. Chance,

T. Gaasterland, D. Lin, A. Sali et al. 1999. Structural genomics: beyond the human genome project. Nat. Genet. 23:151-157.

9.Cassman, M., T. Hunter, and T. Pawson. 2000. Proteins suggest form of their own database. Nature 403:591-592.

10.Celis, J., P. Gromaov, M. Ostergaard, P. Madsen, B. Honore, K. Deigaard, E. Olsen et al. 1996. Human 2-D PAGE databases for proteome analysis in health and disease: http://biobase.dk/gcgibin/celis. FEBS Lett. 398:120-134.

11.Fields, S. 2001. Proteomics in genomeland. Science 291:1221-1224.

12.Fields, S. and O.K. Song. 1989. A novel genetic system to detect protein-protein interactions. Nature 340:245-246.

13.Fung, E.T., V. Thulasiraman, S.R. Weinberger, and E.A. Dalmasso. 2001. Protein chips for differential profiling. Curr. Opin. Biotechnol. 12:65-69.

14.Fung, E.T., G.L. Wright, and E.A. Dalmasso. 2000. Proteomic strategies for biomarker identification: progress and challenges. Curr. Opin. Mol. Ther. 2:643-650.

15.Grandi, G. 2001. Antibacterial vaccine design using genomics and proteomics. Trends Biotechnol. 19:181-188.

16.Gygi, S.P. and R. Aebersold. 2000. Mass spectrometry and proteomics. Curr. Opin. Chem. Biol. 4:489-494.

17.Gygi, S.P., B. Rist, and R. Aebersold. 2000. Measuring gene expression by quantitative proteome analysis. Curr. Opin. Biotechnol. 11:396-401.

18.Gygi, S.P., B. Rist, S.A. Gerber, F. Turecek, M.H. Gelb, and R. Aebersold. 1999. Quantitative analysis of complex protein mixtures using isotope-coded affinity tags. Nat. Biotechnol. 17:994-999.

19.Harris, S. and S.M. Foord. 2000. Transgenic gene knock-outs: functional genomics and therapeutic target selection. Pharmacogenomics 1:433-443.

20.Henzel, W.J., T.M. Billeci, J.T. Stults, and S.C. Wong. 1997. Identifying proteins from two-dimensional gels by molecular mass searching of peptide fragments in protein sequence databases. Proc. Natl. Acad. Sci. USA 90:5011-5015.
21.Holt, L.J., C. Enever, R.M. DeWildt, and I.M. Tomlinson. 2000. The use of recombinant antibodies in proteomics. Curr. Opin. Biotechnol. 11:445-449.

22.International Human Genome Sequencing Consortium. 2001. Initial sequencing and analysis of the human genome. Nature 409:860-921.

23.Jimenez-Sanchez, G., B. Childs, and D. Valle. 2001. Human disease genes. Nature 409:853-855.

24.Li, M. 2000. Applications of display technology in protein analysis. Nat.Biotechnol. 18:1251-1256.

25.Lueking, A., M. Horn, H. Eickhoff, H. Lehrach, and G. Walter. 1999. Protein microarrays for gene expression and antibody screening. Anal. Biochem. 270:103-111.

26.MacBeath, G., and S.L. Schreiber. 2000. Printing proteins as microarrays for high-throughput function determination. Science 289:1760-1763.

27.Marcotte, E.M., M. Pellegrini, H.L. Ng, D.W. Rice, T.O. Yeates, and D. Eisenberg. 1999. Detecting protein function and protein-protein interactions from genome sequences. Science 285:751-753.

28.McCraith, S., T. Holtzman, B. Moss, and S. Fields. 2000. Genome-wide analysis of vaccinia virus protein-protein interactions. Proc. Natl. Acad. Sci. USA 97:4879-4884.

29.Mendelsohn, A.R. and R Brent. 1999. Protein interaction methodstoward an endgame. Science 284:1948-1950.

30.Montelione, G. and S. Anderson. 1999. Structural genomics: keystone for a human proteome project. Nat. Struct. Biol. 6:11-12.

31.O'Donovan, C., R. Apweiler, and A. Bairoch. 2001. The human proteomics initiative. Trends Biotechnol. 19:178-181.

32.O'Farrell, P.H. 1975. High resolution two-dimensional electrophoresis of proteins. J. Biol. Chem. 250:4007-4021.

33.Pandley, A. and M. Mann. 2000. Proteomics to study gene and genomes. Nature 405:837-846.

34.Pawson, T. and P. Nash. 2000. Protein-protein interactions define specificity in signal transduction. Genes Dev. 14:1027-1047.

35.Plowman, G.D., S. Sudarsanam, J. Bingham, D. Whyte, and T. Hunter. 1999. The protein kinases of Caenorhabditis elegans: a model for signal transduction in multicellular organisms. Proc Natl. Acad. Sci. USA 96:13603-13610.

36.Robinson, D.R., Y.M. Wu, and S.F. Lin. 2000. The protein kinase family of the human genome. Oncogene 19:5548-5557.

37.Shevchenko, A., A. Lobada, A. Shevchenko, W. Ens, and K.G. Standing. 2000. MALDI quadrapole time-of-flight mass spectrometry system for automated proteomic research. Anal. Chem. 72:21322141.

38.Uetz, P., L. Giot, G. Cagney, T.A. Mansfield, R.S. Judson, J.R. Knight, D. Lockshon et al. 2000. A comprehensive analysis of protein-protein interactions in Saccharomyces cerevisiae. Nature 403:623-627.

39.Venter, J.C., M.D. Adams, E.W. Myers, P.W. Li, R.J. Mural, G.G. Sutton, H.O. Smith et al. 2001. The sequence of the human genome. Science 291:1304-1351.

40.Vidal, M. and H. Endoh. 1999. Prospects for drug screening using the reverse two-hybrid system. Trends Biotechnol. 17:374-381.

41.Walhout, A.J., R. Sordella, X. Lu, L. Hartley, G.F. Temple, M.A. Brasch, N. Thierry-Mieg, and M. Vidal. 2000. Protein interaction mapping in C. elegans using proteins involved in vulval development. Science 287:116-122.

42.Wilkins, M.R., C. Pasquali, R.D. Appel, K. Ou, O. Golaz, J.C. Sanchez, J.X. Yan et al. 1996. From proteins to proteomes: largescale identification by two-dimensional electrophoresis and amino acid analysis. Biotechnology 14:61-65.

43. Wuthrich, K. 1998. The second decade-into the third millennium. Nat. Struct. Biol. 5:492-495.

Suggestions for contributions to the "Drug Discovery and Genomic Technologies" section are welcomed by its editor, Dr. Tomi K. Sawyer (tomi.sawyer@ariad.com) 